\title{
2 Elephant Genomes Elucidate Disease Defenses and Other
}

\section{Traits}

4 M. Harrison ${ }^{2,6}$, Wendy K. Kiso ${ }^{7}$, Dennis L. Schmitt ${ }^{7,8}$, Michael M. Garner ${ }^{9}$, C. Athena Aktipis ${ }^{2,10}$, Carlo C. Maley 2,5 , Amy M. Boddy 2,11 , Mark Yandell ${ }^{4}$, Christopher Gregg ${ }^{3}$, Joshua D. Schiffman ${ }^{2,12,13}$, Lisa M. Abegglen ${ }^{2,12}$

${ }^{1}$ School of Informatics, Computing, and Cyber Systems, Northern Arizona University, Flagstaff, AZ; ${ }^{2}$ Arizona Cancer Evolution Center, Arizona State University, Tempe, AZ; ${ }^{3}$ Department of Neurobiology and Anatomy, University of Utah, Salt Lake City, UT 84132-3401, USA; ${ }^{4}$ Department of Genetics, University of Utah, Salt Lake City, UT, USA; ${ }^{5}$ Center for Biocomputing, Security and Society, Biodesign Institute, Arizona State University, Tempe, USA; ${ }^{6}$ Department of Clinical Sciences, North Carolina State University, Raleigh, NC, 27607 USA; ${ }^{7}$ Ringling Bros Center for Elephant Conservation, Polk City, Florida USA; ${ }^{8}$ William H. Darr College of Agriculture, Missouri State University, Springfield, Missouri, USA; ${ }^{9}$ Northwest ZooPath, Monroe, WA 98272; ${ }^{10}$ Arizona State University, Department of Psychology, Tempe, AZ; ${ }^{11}$ Department of Anthropology, University of California, Santa Barbara CA, USA; ${ }^{12}$ Department of Pediatrics \& Huntsman Cancer Institute, University of Utah, Salt Lake City, UT, USA; ${ }^{13}$ PEEL Therapeutics, Inc., Salt Lake City, UT, USA \& Haifa, IL

*Authors contributed equally

23 Authors for correspondence: Email: $\underline{\text { marc.tollis@nau.edu and lisa.abegglen@hci.utah.edu }}$ 


\section{Abstract}

2 Disease susceptibility and resistance comprise important factors in conservation, particularly in

3 elephants. To determine genetic mechanisms underlying disease resistance and other unique

4 elephant traits, we estimated 862 and 1,017 potential regulatory elements in Asian and African

5 elephants, respectively. These elements are significantly enriched in both species with

6 differentially expressed genes involved in immunity pathways, including tumor-necrosis factor

7 which plays a role in the response to elephant endotheliotropic herpesvirus (EEHV). Population

8 genomics analyses indicate that amplified TP53 retrogenes are maintained by purifying

9 selection and may contribute to cancer resistance in elephants, including less malignancies in

10 African vs. Asian elephants. Positive selection scans across elephant genomes revealed genes

11 that may control iconic elephant traits such as tusk development, memory, and somatic

12 maintenance. Our study supports the hypothesis that interspecies variation in gene regulation

13 contributes to differential inflammatory responses leading to increased infectious disease and

14 cancer susceptibility in Asian versus African elephants. Genomics can inform functional

15 immunological studies which may improve both conservation for elephants and human

16 therapies. 


\section{Introduction}

2 Elephants (family Elephantidae) first appeared on the planet $\sim 5-10$ million years ago (MYA) and

3 three species roam today: the African bush elephant (Loxodonta africana), the African forest

4 elephant. (L. cyclotis), and the Asian elephant (Elephas maximus). These species are the only

5 surviving members of the once diverse proboscidean clade of afrotherian mammals ${ }^{1}$. Other

6 elephantids such as straight-tusked elephants (genus Paleoloxodon) and mammoths

7 (Mammuthus) went extinct around 34,000 and 4,300 years ago, respectively ${ }^{2,3}$. All extant

8 elephant species are now threatened with extinction, largely due to poaching and habitat loss.

9 Asian elephants are "endangered," with only $\sim 200$ wild individuals in some countries ${ }^{4}$, and

10 African elephants are "vulnerable" with only $\sim 400,000$ wild individuals after a decrease of

$11 \sim 100,000$ individuals between 2007 and $2016^{5,6}$. It is imperative that we study the genetics of

12 these amazing creatures and how this knowledge of their evolutionary history can contribute to

13 their continued conservation.

Elephants share many charismatic traits such as prehensile trunks, ivory tusks,

intelligence with long-term memory, and large body sizes ${ }^{1}$. Given their long lifespans of nearly

80 years for Asian $^{7}$ and approximately 65 years for African elephants ${ }^{8}$, coupled with lengthy

17 gestation periods of 22 months, disease defense has evolved as an important trait for elephants. Differences in disease susceptibility between species have urgent ramifications for elephant conservation ${ }^{9}$. In addition to poaching, Asian elephants are threatened by an acute

20 hemorrhagic disease resulting from infection with elephant endotheliotropic herpesvirus

$21(E E H V)^{10,11}$. While fatalities in African elephant calves from EEHV also have been reported,

22 mortality rates are higher for Asian elephants suggesting a genetic component for increased

23 EEHV lethality. Asian elephants also are more susceptible to tuberculosis (TB) infection

24 (Mycobacterium tuberculosis and M. bovis) $)^{9}$ (Fisher's Exact Test, $\mathrm{P}=2.52 \mathrm{e}-04$; Chi-squared test, $25 \mathrm{P}=6.84 \mathrm{e}-04$; Supplementary Fig. 1). Understanding the functional immunological and molecular 26 basis of disease response in elephants may improve their conservation and medical care. 
Another important disease for elephants is cancer, albeit for different reasons. Elephant

2 cancer mortality rates are low compared to humans, despite the fact that cancer is a body size-

3 and age-related disease ${ }^{12}$. A potential mechanism of cancer resistance for elephants is an

4 enhanced apoptotic response of elephant cells to DNA damage associated with extensive

5 amplification of retrogene copies of the tumor suppressor gene $T P 53^{12-14}$, yet there are still

6 unknowns related to cancer in elephants. For instance, it is unclear if variation in TP53 copy

7 number contributes to differences in cancer defense between elephant species. Also, it is not

8 known whether the observed differential responses to EEHV and TB between Asian and African

9 elephants relate to cancer susceptibility. Detailed analyses of cancer prevalence and mortality in

10 elephants may provide insight into how elephants evolved to handle disease. Here, we add to

11 the knowledge of elephant cancer prevalence with data from zoos accredited by the Association

12 of Zoos and Aquariums (AZA). AZA sets the standards for animal care and welfare in the United

13 States (https://www.aza.org/about-us, last accessed September 2020). Every elephant in an

14 AZA facility undergoes routine blood screens, full body examinations, and thorough necropsy

15 upon death, making the likelihood of documenting elephant cancer high.

In addition to maintaining the health of elephants under human care to improve breeding

17 and species survival plans, conservation efforts can benefit from genomic studies that identify

18 genetic variants associated with traits such as disease defense ${ }^{15}$. However, the few elephant

19 functional genomic studies currently available are limited to a small number of individuals and

20 species $^{16-19}$ and the genetic etiologies of most elephant traits are unknown. In our study, we

21 analyze data from three living and two extinct species in comparative and population genomic

22 frameworks in order to understand the genomic basis of elephant traits, including what drives

23 different disease outcomes between species. 


\section{Results}

\section{Asian elephants suffer from higher rates of malignant cancers than African elephants}

3 To estimate rates of neoplasia and malignancy in elephants, we collected and analyzed

4 pathology data from 26 AZA-accredited zoos in the USA, which included diagnoses from 76

5 different elephants ( $n=35$ African and $n=41$ Asian). We found that $5.71 \%$ of the African

6 elephants and $41.46 \%$ of the Asian elephants were diagnosed with neoplasia (which included

7 benign and malignant tumors) (Fisher's Exact Test, $P=3.78 e-04$; Chi-square test, $P=8.95 e-04$ )

8 (Table 1, Supplementary Table 1). Sixty-nine percent of elephant tumors were benign, and

$914.63 \%$ of Asian elephants were diagnosed with malignant tumors compared to zero in African

10 elephants (Fisher's Exact Test, $\mathrm{P}=0.028$; Chi-squared test, $\mathrm{P}=0.053$ ). In contrast, the lifetime

11 risk of developing malignant cancer is $39.5 \%$ for humans ${ }^{20}$ and the lifetime risk of developing

12 benign tumors is even higher, with $70 \%-80 \%$ of women developing uterine fibroids

13 (leiomyomas) alone ${ }^{21}$. Asian elephants are also reported to have a high prevalence of uterine

14 leiomyomas ${ }^{22}$, including seven in our dataset. Our results confirm that (1) malignant cancer

15 rates in elephants are lower than in humans and (2) Asian elephants are diagnosed with both

16 neoplasia and malignancies more often than African elephants in zoos. 
1 Table 1: Cancer diagnoses and prevalence in African and Asian elephants.

\begin{tabular}{|c|c|c|c|c|c|}
\hline \multicolumn{6}{|c|}{ Neoplasia and Malignant Prevalence } \\
\hline \multirow{2}{*}{ Species } & \multirow{2}{*}{ Total Individuals } & Neoplasia & Neoplasia & Malignant & Malignant \\
\hline & & Cases & $\%$ & Cases & $\%$ \\
\hline Asian elephant & 41 & 17 & 41.46 & 6 & 14.63 \\
\hline African elephant & 35 & 2 & 5.71 & 0 & 0 \\
\hline Total elephants & 76 & 19 & 25 & 6 & 7.89 \\
\hline \multicolumn{6}{|c|}{ Neoplasia Diagnoses in African/Asian Elephants } \\
\hline Species & Sex & Age & Lesion Type & Lesion Site & Malignant \\
\hline African elephant & Female & 28 & Polyp & Vagina & No \\
\hline African elephant & NA & NA & Adenoma & Parathyroid & No \\
\hline Asian elephant & Female & 45 & Polyp & Vulva & No \\
\hline Asian elephant & Female & $50 ; 50$ & $\begin{array}{l}\text { Polyp; } \\
\text { Leiomyoma }\end{array}$ & Uterus;Uterus & No;No \\
\hline \multirow{2}{*}{ Asian elephant } & \multirow{2}{*}{ Female } & \multirow{2}{*}{$30 ; 40$} & Polyp; & Vagina; & \multirow{2}{*}{ No } \\
\hline & & & $\begin{array}{l}\text { Spindle Cell } \\
\text { Tumor }\end{array}$ & Leg & \\
\hline Asian elephant & Female & 39 & Leiomyoma & Uterus & No \\
\hline Asian elephant & Female & 39 & Mast Cell Tumor & Abdomen & No \\
\hline
\end{tabular}




\begin{tabular}{|c|c|c|c|c|c|}
\hline Asian elephant & Male & 35 & Papilloma & Trunk & No \\
\hline Asian elephant & Female & 50 & Papilloma & Skin & No \\
\hline Asian elephant & Female & 36 & Papilloma & Skin & No \\
\hline Asian elephant & Female & 50 & Adenocarcinoma & Breast & Yes \\
\hline Asian elephant & Female & $59 ; 59$ & $\begin{array}{l}\text { Adenocarcinoma; } \\
\text { Leiomyoma }\end{array}$ & Uterus; Uterus & Yes; No \\
\hline \multirow{7}{*}{$\begin{array}{c}\text { Asian elephant } \\
\text { (7) }\end{array}$} & \multirow{7}{*}{ NA } & \multirow{7}{*}{ NA } & $\begin{array}{l}\text { Adenocarcinoma } \\
\text { (2); }\end{array}$ & Uterus (2); & \multirow{4}{*}{ Yes } \\
\hline & & & $\begin{array}{l}\text { Undifferentiated } \\
\text { Malignant } \\
\text { Neoplasm (1); }\end{array}$ & Uterus (1); & \\
\hline & & & $\begin{array}{l}\text { Leiomyosarcoma } \\
\text { (1); }\end{array}$ & Lung (1); & \\
\hline & & & Sarcoma (1) & Liver (1) & \\
\hline & & & Leiomyoma (4); & Uterus (4); & \multirow{3}{*}{ No } \\
\hline & & & Leiomyoma (1); & Stomach (1); & \\
\hline & & & Microadenoma (1) & $\begin{array}{l}\text { Pituitary } \\
\text { Gland (1) }\end{array}$ & \\
\hline
\end{tabular}

2 Elephant-specific accelerated genomic regions are enriched for immune pathways and 3 correlate with species-specific gene expression patterns

4 To explore the genomic mechanisms governing disease response and other traits across

5 elephant species, we sequenced and assembled the genome of an Asian elephant (“Icky") born

6 in Myanmar and currently under human care at $94.4 \mathrm{X}$ coverage with a final scaffold N50 of 2.77

7 Mb (GCA_014332765.1) (Supplementary Information, Supplementary Table 2, Supplementary 
1 Table 3, Supplementary Table 4). We also improved the African bush elephant genome

2 assembly with Hi-C libraries (Supplementary Information, Supplementary Fig. 2, Supplementary

3 Table 5). These genome assemblies were used to generate a whole genome alignment (WGA)

4 with 10 other mammals, which we used to define accelerated genomic regions (ARs) unique to

5 Asian and African elephants. We first defined 676,509 60 bp regions that were present in Asian

6 and African elephants and conserved in the 10 background species with phastCons ${ }^{23,24}$

7 (conserved regions or CRs, Fig. 1a).

$8 \quad$ Asian and African elephants likely diverged $\sim 5 \mathrm{MYA}^{25}$, and since differences between

9 closely related mammals are primarily due to changes in non-coding regulatory genomic

10 regions ${ }^{24,26,27}$, we focused on the 376,899 CRs detected in non-coding regions. We tested these

11 for accelerated substitution rates in elephants with phyloP ${ }^{24,26}$ and found 3,622 regions with

12 significantly increased nucleotide substitution rates in the Asian elephant while 3,777 regions

13 were accelerated in the African bush elephant (q-value < 0.10). We found 2,418 ARs shared

14 between both species, with 862 Asian elephant-specific and 1,017 African bush elephant-

15 specific ARs (Fig. 1b).

ARs common to Asian and African bush elephants were likely driven by changes pre-

17 dating the evolutionary divergence of the two elephants, while Asian elephant- and African bush

18 elephant-specific ARs may point to enhancers driving gene expression level changes that

19 impact phenotypes distinguishing the two species. Using available African bush elephant and

20 Asian elephant peripheral blood mononuclear cell (PBMC) RNA-Seq data ${ }^{28,19}$, we defined 5,034

21 differentially expressed (DE) elephant genes (false discovery rate or FDR < 0.01). Both Asian

22 elephant- and African bush elephant-specific ARs were significantly enriched near DE genes

23 relative to $\mathrm{CRs}$ (Fisher's Exact Test, $\mathrm{P}=2.05 \mathrm{e}-4, \mathrm{P}=8.30 \mathrm{e}-7$, respectively). Meanwhile, the 2,418

24 ARs common to both elephants were not significantly enriched near DE genes (Fig. 1c). This

25 pattern remained robust with subsets of increasingly significantly DE genes based on adjusted

26 p-values (Supplementary Fig. 3). Asian elephant- and African bush elephant-specific ARs 
disproportionately overlapped DE gene regulatory regions relative to the common ARs (Chi-

2 squared test, $P=0.019$ and $P=0.001$ respectively; Fig. 1d, Fig. 1e), suggesting that some ARs

3 reflect changes in regulatory regions that alter gene expression patterns in elephant PBMCs.

4 Figure 1. Elephant accelerated regions. Using a whole genome alignment of 12 mammals (a), we defined genomic regions that were accelerated (ARs) in two elephant species (red), yet conserved in the set of background species (blue). Branch lengths are given in terms of mean substitutions per site at fourfold degenerate sites (neutral model). Among the ARs detected in elephants, we found ARs common to both elephant species as well as ARs specific to either Asian or African bush elephants (b). Differentially expressed (DE) genes were much more likely to be found in Asian elephant-specific (Fisher's Exact Test, $\mathrm{P}=2.05 \mathrm{e}-4$ ) and African elephantspecific ( $P=8.30 \mathrm{e}-7)$ ARs than in common ARs (c). Species-specific ARs disproportionately overlap DE gene regulatory regions relative to the common ARs (Chi-squared test, $p=0.019$ and $13 \mathrm{p}=0.001$ respectively) $(d, e)$.

a

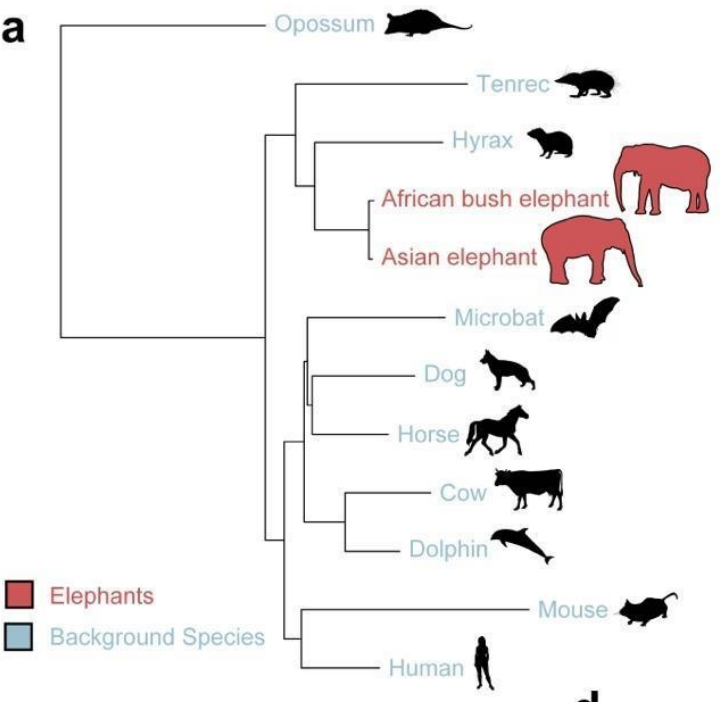

C

Elephant DE Gene AR Enrichment

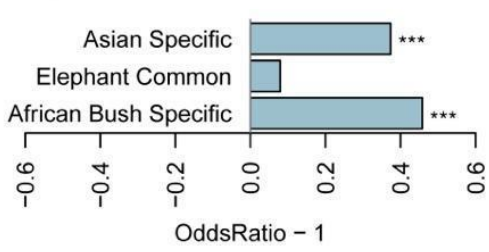

b

\section{Noncoding Elephant AR Overlap}

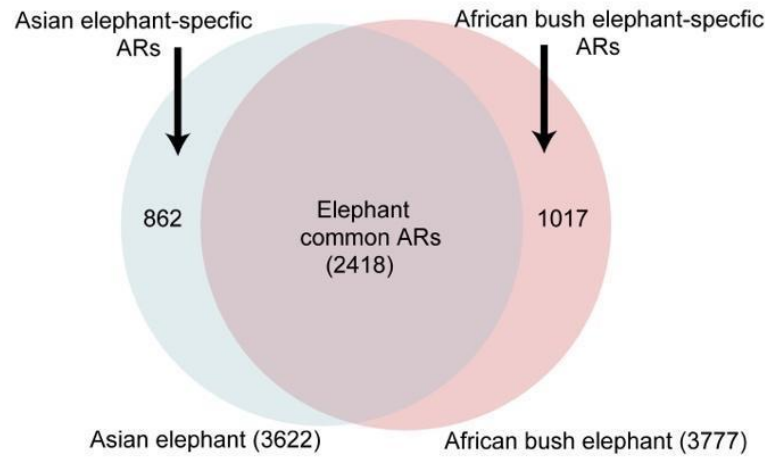

We functionally annotated AR contributions to African and Asian elephant species 
1 test that compared general linearized models (see Materials and Methods), 605 out of 607

2 (99.6\%) of GO terms were uniquely enriched in the elephant ARs in contrast to ARs found in

3 other mammalian lineages ${ }^{19}$. This suggests that the enrichment of GO terms in the elephant

4 ARs are significant in elephants in contrast to other mammals. Of 18,056 BP GO terms, 252

5 were significantly enriched in Asian elephant specific ARs and 275 were enriched in African

6 elephant specific ARs (q-value < 0.05). Many of the GO terms related to the immune system in

7 both elephant species (Fig. 2; Supplementary Fig. 4; Supplementary Fig. 5; Supplementary

8 Data). The broad term 'immune system process' was 4.5 and 2.8 fold enriched with Asian and

9 African elephant-specific ARs (q-value $=4.87 \mathrm{e}-12$ and $7.84 \mathrm{e}-06$, respectively), but not

10 significantly enriched with elephant common ARs. Our results suggest (1) many of the species-

11 specific ARs alter gene expression patterns and transcription factor binding networks that

12 eventuate differences in immune function, and (2) Asian-elephant ARs are more enriched in

13 immune pathways than African elephant-specific ARs in terms of both fold-enrichment and

14 statistical significance.

15 We found 109 GO terms significantly enriched with elephant common ARs (q-value <

160.05 , Supplementary Fig. 6, Supplementary Data), many of which were related to cancer,

17 including "sphingolipid metabolic process" which was in the top 10 most significantly enriched

18 GO terms for both elephant common (5.7 fold enrichment, q-value $=4.69 \mathrm{e}-08$ ) and African

19 elephant-specific (17.3 fold enrichment, q-value = 4.18e-22) ARs (Fig. 2). Sphingolipid

20 metabolites mediate the signalling cascades involved in apoptosis ${ }^{29}$, necrosis ${ }^{29}$, senescence ${ }^{30}$,

21 and inflammation ${ }^{31}$. We found 2.9 and 3.6 fold enrichments for 'tumor necrosis factor (TNF)-

22 mediated signaling pathway' (q-value=4.75e-04) and 'positive regulation of TNF production' (q-

23 value $=1.75 \mathrm{e}-03$ ) in the common ARs, and a 21.5 fold enrichment of "negative regulation of

24 TNF secretion" in African elephant-specific ARs (q-value = 5.01e-04). TNF is a cytokine involved

25 in cell differentiation and death that can induce the necrosis of cancer cells ${ }^{34}$. The upregulation 
1 of TNF-alpha has been associated with increased apoptosis in EEHV-infected Asian elephant

2 PBMCs as well ${ }^{11}$.

3 In a check for reproducibility, we found that the number of African elephant-specific ARs

4 assigned to each gene was correlated with previous studies ${ }^{19}(R=0.82)$. The gene most

5 enriched with previously defined non-coding African elephant ARs was the DNA repair gene

6 FANCL (7.3 fold enrichment; q-value $=2.16 \mathrm{e}-56)$, which mediates the E3 ligase activity of the

7 Fanconi anemia core complex, a master regulator of DNA repair ${ }^{32}$. We found that FANCL was

8 the third most significantly enriched gene in both African and Asian elephant ARs relative to

9 CRs with 4.6 and 4.9 fold enrichments (q-value $=1.27 \mathrm{e}-14$ and $4.46 \mathrm{e}-16$ ). Of 50 African

10 elephant ARs and 51 Asian elephant ARs assigned to FANCL, 43 are common to both elephant

11 species suggesting their acceleration predates African-Asian elephant divergence. These

12 results suggest non-coding cis-regulatory elements have regulated cancer resistance

13 adaptations throughout elephant evolution, particularly in the ancestor of modern elephants and

14 the lineage leading to the African bush elephant. 
1 Figure 2. Top 10 Biological Process gene ontology (GO) terms most significantly 2 enriched with elephant accelerated genomic regions (ARs). The 10 most significantly 3 enriched GO terms in terms of -log10(q-value) for each set of ARs (African elephant-specific, 4 African and Asian elephant common, Asian elephant-specific), and their overlap. "Innate immune response" and "immune system process" are in the top 10 most significantly enriched GO terms for Asian elephant-specific ARs, are significantly enriched in African elephant specific ARs but not in the top 10, and are not significantly enriched in the common ARs. "Negative 9 elephant-specific ARs.

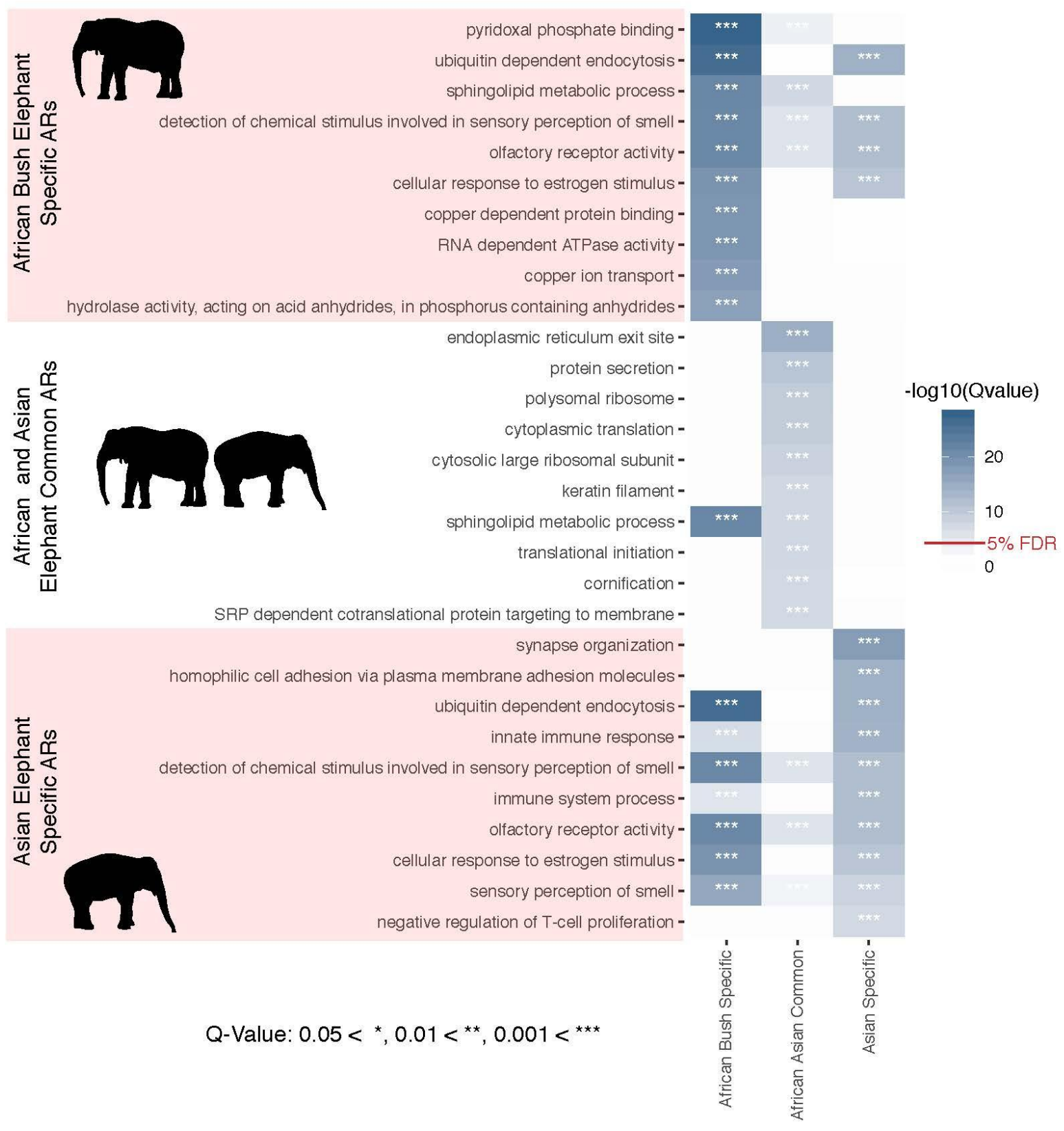




\section{Evolution of TP53 and its retrogenes in elephant genomes}

2 The enhanced apoptotic response to DNA damage in elephant cells correlates with the expansion of $\sim 20$ copies of the tumor suppressor gene TP53 in elephant genomes ${ }^{12-14}$, and we wanted to (1) understand the evolutionary origins of TP53 gene duplications in Asian and African bush elephants and (2) determine if TP53 copy number is related to body size evolution in elephants. We annotated TP53 homologs in 44 mammalian genomes including Icky the Asian elephant, an additional genome assembly of an Asian elephant ${ }^{33-35}$ ("Methai”, born in Thailand and living at the Houston Zoo, assembly available at www.dnazoo.org, last accessed September 2020), and the African bush elephant assembly presented in this study (Supplementary Table 6), and incorporated them in a molecular clock analysis. We estimated that TP53 retrogene copies originated in the paneungulate ancestor of manatees and elephants 55-60 million years ago (MYA) (41.3-75.2 95\% highest posterior density or HPD) (Fig. 3a,

13 Supplementary Fig. 7). A subsequent TP53 expansion began 45 MYA (30.7-60.1 95\% HPD)

14 in a common ancestor of Asian and African elephants, and continued throughout elephantid

15 evolution. We estimated 19 copies of TP53 in the African bush elephant genome assembly, and

9-11 TP53 copies in the Asian elephant genome based on the two assemblies for the species.

We mapped whole genome shotgun data from multiple individuals belonging to three

living and two extinct elephant species (Supplementary Table 7) to the bush elephant genome annotation (loxAfr3) and used normalized read counts to estimate TP53 copy numbers in elephant genomes (Figure 3b; Supplementary Table 8). Based on read depth, African bush

21 elephants $(n=4)$ have on average $\sim 19-23$ TP53 copies in their genomes, and Asian elephant

22 genomes $(n=7)$ contain as few as 10 TP53 copies, or as many as 37, but without these outliers

23 average $\sim 19-22$ TP53 copies in their genomes. These estimates are similar to previous

24 estimates of TP53 copy numbers for bush and Asian elephants based on smaller numbers of 25 individuals ${ }^{12-14}$. We estimated $\sim 21-24$ TP53 copy number variants in forest elephant genomes $26(n=2)$. The woolly mammoths $(n=2)$ were estimated to have between 19 and 28 TP53 copies in 
1 their genomes, which was slightly higher than previous estimates ${ }^{14}$. Meanwhile, the straight-

2 tusked elephant genome contained 22-25 TP53 copies.

3 The number of TP53 copies estimated in the genomes of Asian elephants differed based

4 on the method used. For instance, we validated two TP53 retrogenes in Icky's genome

5 assembly, which phylogenetically clustered closely with two of the nine retrogenes validated in

6 Methai's genome assembly (Fig. 3a). Taken together, based on the reciprocal BLAT searches

7 of both assemblies we estimated 9-11 TP53 copies in the Asian elephant genome. However,

8 based on normalized read counts, we estimated 10-37 TP53 copies (Fig. 3b), which is more

9 consistent with previous studies ${ }^{12,14}$. The lower estimates we obtained from the Asian elephant

10 genome assemblies may be due to poorly resolved repetitive regions which hamper graph-

11 based de novo genome assemblers ${ }^{33}$. Subsequent refinement of Asian elephant genome

12 assemblies using long read sequencing may better resolve these regions. In the meantime, our

13 results suggest that copy number estimates based on read depth are useful approximations for

14 approaches validated from genomic DNA. 
Figure 3. Evolution of TP53 in elephants and other afrotherians. (a) Phylogeny of TP53 sequences extracted from afrotherian genomes. TP53 retrogenes (Manatee RG1, eleMax, and loxAfr) appeared early in the evolution of paenungulates $~ 55-60$ million years ago (MYA), followed by subsequent amplification in the elephant lineage $~ 45$ MYA. Red dots indicate estimated nodes with posterior probability $\geq 90 \%$. Red eleMax indicates TP53 retrogene sequences extracted from the "Methai" Asian elephant assembly, and blue eleMax indicates TP53 retrogene sequences extracted from the Asian elephant assembly "Icky" presented in this study. (b) TP53 copy number estimates based on read counts from three living elephants (African bush elephant ( $\mathrm{n}=4$, minimum 18, maximum 22.4, median 20.1, 25th percentile 19.3, 75th percentile 20.8 ), forest elephant $n=2$, minimum 24.3 , maximum 25.2 , median 24.7 , 25th percentile $24.5,75$ th percentile 25 ) and Asian elephant ( $n=7$, minimum 10.9 , maximum 36.8 , median 21.9 , 25th percentile 18.5, 75th percentile 23.4)) and two extinct (straight-tusked $(n=1$, 25.1 ) and woolly mammoth $(n=2$, minimum 21 , maximum 28 , median 24.51 , 25 th percentile 22.8, 75th percentile 26.3)) elephant species. Shoulder height estimates from Larramendi et al. (2015). Phylogeny is schematic only and represents relationships from Palkopuolou et al.

16 (2018).
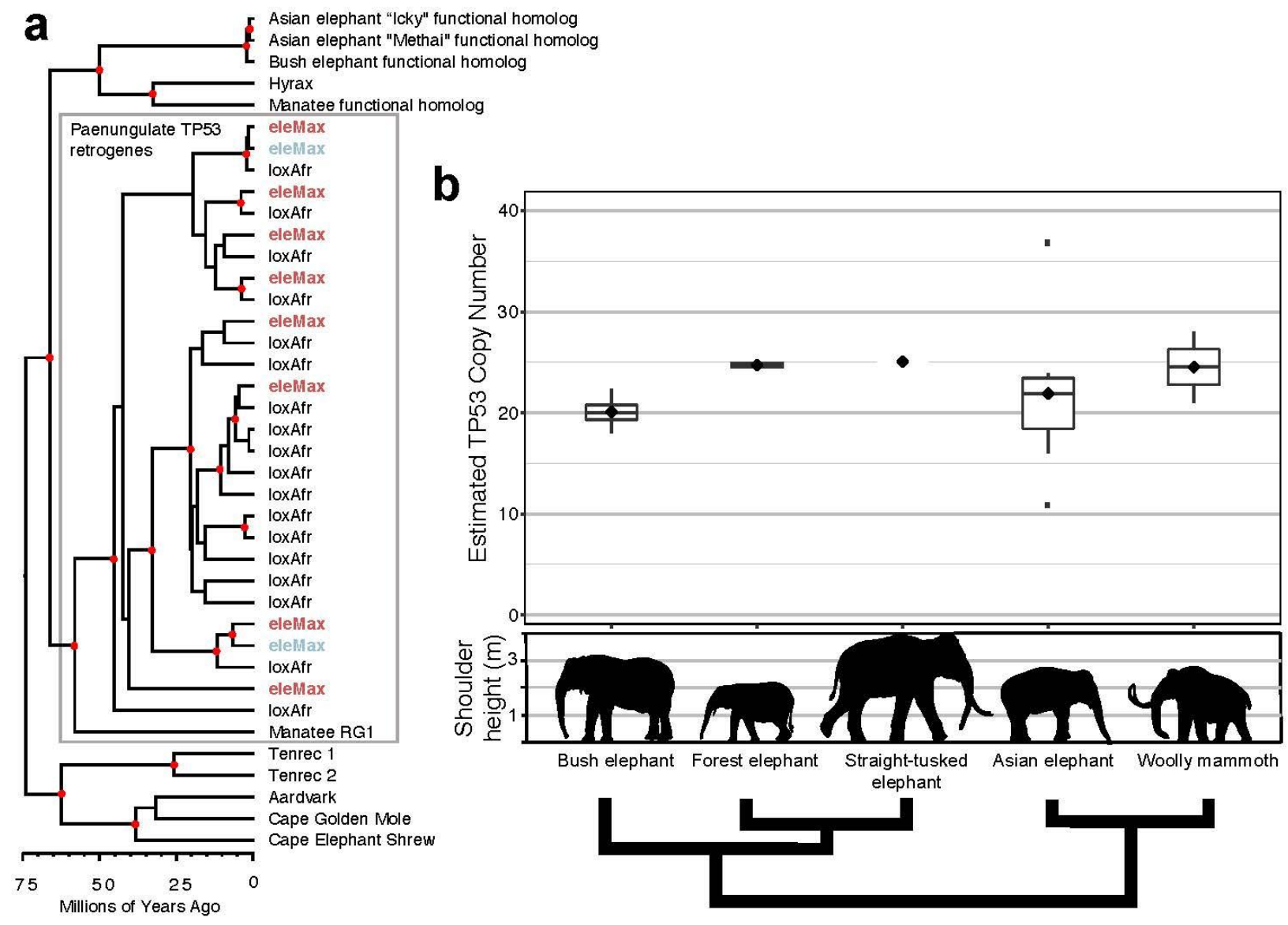


\section{A bottleneck for bush elephants and a deep divergence for forest elephants}

2 Our next goals were to (1) determine if TP53 paralogs segregate as functional alleles in

3 elephant populations and (2) estimate regions of adaptive evolution (positive selection) that may

4 control phenotypes in modern elephants. To do this, we utilized the aligned genomic sequences

5 from the living elephant species to call variants with freebayes v1.3.1-12 ${ }^{36}$, genotyping

$641,296,555$ biallelic single nucleotide polymorphisms (SNPs), averaging one SNP every 77

7 bases and with a genome-wide transition-transversion ratio of 2.46. Altogether, we annotated

8290,965 exonic, 11,245,343 intronic, and 32,512,650 intergenic SNPs across the 13 elephant

9 genomes.

To establish the neutral background against which to compare putative adaptively evolving regions of the genome, we assessed the demographic history of each elephant species with summary population genomics statistics. Among the three living species of elephants, bush

13 elephants averaged the lowest nucleotide diversity (0.0008, s.d. 0.001$)$, followed by Asian

14 elephants (0.0011, s.d. 0.001) and forest elephants (0.002, s.d. 0.002) (Fig. 4a). The distribution

15 of Tajima's $\mathrm{D}$ in $10 \mathrm{~Kb}$ genomic bins calculated for bush elephant revealed an excess of

16 negative values relative to other elephant species (Fig. 4b). We also found a larger proportion

17 of heterozygous sites ( 0.18 and 0.21$)$ in forest elephant genomes compared to all other elephants (Fig. 4c), consistent with the deep genomic divergence reported in this species ${ }^{25,37}$.

After identifying runs of homozygosity $(\mathrm{RoH})$ in each elephant (Fig. 4d, Supplementary

Fig. 8a), we estimated the average inbreeding coefficient $\left(F_{R \circ H}\right)$ to be higher in bush elephants

$21(2.04 \%)$ than Asian (1.64\%) and forest (0.61\%) elephants (Supplementary Fig. 8b). Taken

22 together, the excess of low frequency polymorphisms, $\mathrm{RoH}$, and $F_{\mathrm{ROH}}$ suggest a strong

23 population bottleneck in bush elephants. The Asian elephant from Borneo contained the

24 smallest number of heterozygous genotyped sites among all elephants (0.03), and the highest

$25 F_{R o H}(5.39 \%)$, consistent with recent analyses showing that the Borneo subpopulation of $E$.

26 maximus is genetically isolated ${ }^{25}$. 
Figure 4. Summary population genomics statistics for three living elephant species. We analyzed $10 \mathrm{Kbp}$ bins for (a) nucleotide diversity and (b) Tajima's D for Asian (Elephas maximus), bush (Loxodonta africana), and forest (L. cyclotis) elephants. (c) Per-individual heterozygosity for Asian, bush, and forest elephants. (d) Number of detected runs of homozygosity for each individual elephant.

a

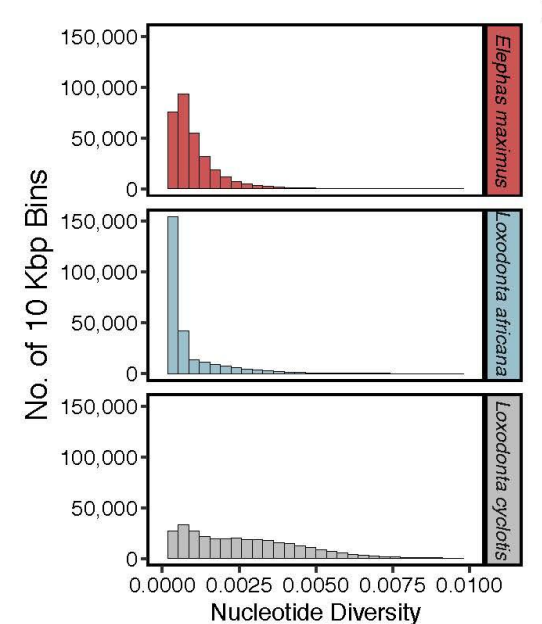

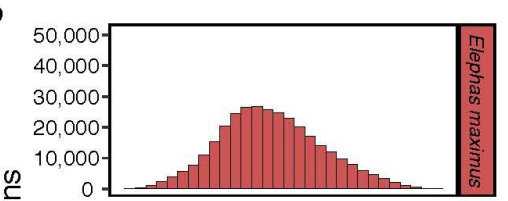
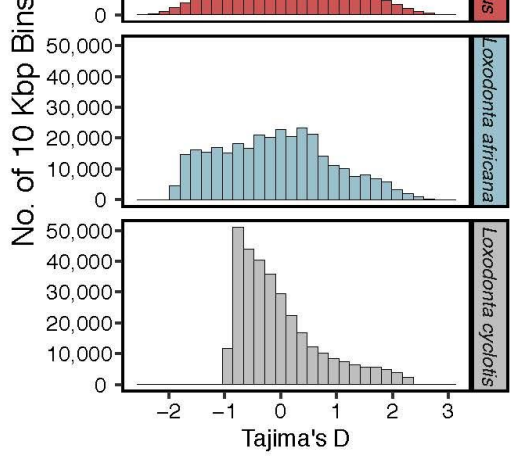

Loxodonta cyclotis
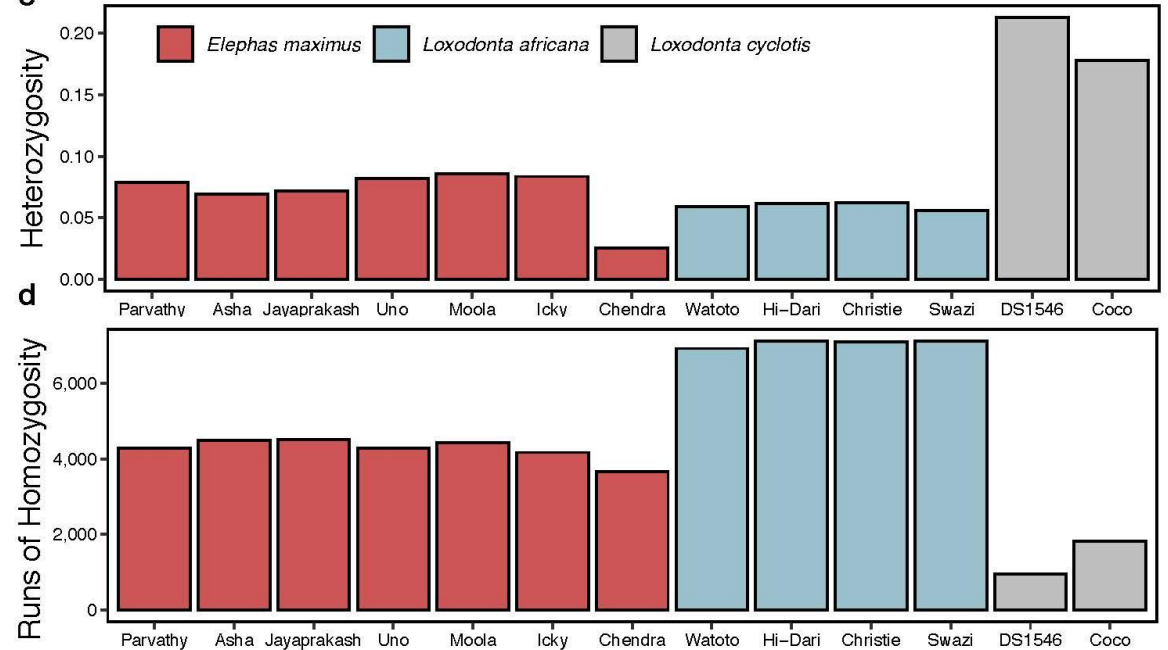

Genetic variation in TP53 copy number variants suggests maintenance of some by purifying selection

We found a high degree of sequence conservation in the TP53 paralogs both within and between the three living elephants (Supplementary Table 9). For instance, the proportion of polymorphic sites in putatively neutrally evolving ancestral repeats was 0.013 , but across 12 annotated TP53 paralogs was 0.004. Despite the deep genomic divergence of forest elephants, we found very little genetic variation in TP53 paralogs for the species, with just a single 
1 segregating site in three of the retrogenes. Across all species, we collected zero

2 nonsynonymous SNPs for the functional homolog (ENSLAFG00000007483), consistent with

3 strong purifying selection on this gene and ENSLAFG00000028299, or "retrogene 9", whose

4 protein expression is stabilized by DNA damage in human cells ${ }^{12}$.

$5 \quad$ We annotated variants in 12 TP53 paralogs based on the bush elephant genome

6 annotation and found few variants affecting gene function (Supplementary Table 10), consisting

7 of mostly missense mutations. There were no variants of high or moderate impact on gene

8 function annotated in the functional homolog (ENSLAFG00000007483), with the majority of

9 variants occurring downstream, in introns, or upstream of the gene. The high degree of

10 sequence conservation across three species of elephant, and in particular the lack of variants

11 with functional effect, especially in "retrogene 9," suggests that at least some TP53 retrogenes

12 are being maintained by purifying selection.

\section{An elephant never forgets: positive selection in living elephants}

14 To assess the impact of natural selection across elephant genomes and its impact on

15 phenotypes, we scanned the genomes of the three extant species for positive selection using

16 SweeD v3.3.1 $1^{38,39}$, hypothesizing that genetic pathways controlling elephant traits would be

17 subjected to selective sweeps. This yielded 24,394 selectively swept outlier regions meeting our

18 statistical thresholds based on neutral expectations (see Materials and Methods) in Asian

19 elephants, which comprise $\sim 0.07 \%$ of the genome and overlapped with 1,611 gene annotations.

20 Out of the 41,204 regions experiencing putative selective sweeps in bush elephants $(\sim 1.3 \%$ of

21 the genome), we detected 2,882 protein-coding genes. We estimated 4,099 protein-coding

22 genes involved in the 51,249 regions involved in putative selective sweeps in forest elephants

$23(\sim 1.6 \%$ of the genome).

24 We found 242 protein-coding genes that overlapped regions with evidence of positive 25 selection and were shared in all three of the living elephant species, which are enriched in BP 
1 GO terms that shed light on the genomic mechanisms controlling many iconic elephant traits.

2 For instance, the most significantly enriched BPs (in terms of fold enrichment) were "dendrite

3 self-avoidance" (27-fold enrichment, FDR=0.02), "ionotropic glutamate receptor signaling

4 pathway" (17-fold enrichment, FDR=0.02), and "regulation of NMDA receptor activity" (14-fold

5 enrichment, FDR=0.03). Many significantly enriched GO terms clustered semantically with

6 "trans-synaptic signaling" (Fig. 5a). We also found significant protein interactions among outlier

7 genes (75 observed edges versus 45 expected; enrichment $\mathrm{P}=3.68 \mathrm{E}-5$ ), including an

8 enrichment of genes associated with the glutamatergic synapse pathway $(7$ of 98 genes,

9 FDR=0.015). Glutamate is the major excitatory neurotransmitter for mammalian nerve cells,

10 mediating excitatory synaptic transmission ${ }^{40}$. These results suggest strong selection in

11 elephants on pathways involved in memory, learning, and the formation of neural networks. The

12 recognition, storing and retrieving of information in the human brain occurs in the temporal lobe,

13 and the temporal lobes of elephants' brains are relatively larger than those of humans, as well

14 as denser and more convoluted ${ }^{41}$. Retrieving information is likely crucial for elephants to find

15 resources across vast and complicated landscapes ${ }^{1}$.

Our genomic results suggest that positive selection has acted on genes involved in

17 elephant tusk formation. Unlike most tusked mammals which feature elongated canines, the elephant tusk is a highly modified upper incisor ${ }^{1}$. We found that the most significantly enriched mouse phenotypes among genes overlapping selective sweeps were "abnormal upper incisor

20 morphology" (FDR=0.001) and "long upper incisors" (FDR=0.003). These included two genes:

21 ANTXR1 and NFIC, (Figure 5b) which are involved in tooth development in humans ${ }^{42-44}$ and 22 mice ${ }^{45}$, respectively.

23 Other significant gene ontology terms from outlier regions in our selective sweep

24 analysis were related to cancer, including cell adhesion (9-fold enrichment, FDR=0.007), cell-

25 cell signaling (3-fold enrichment, FDR=0.01), and cell communication (2-fold enrichment,

26 FDR=0.0001). Significant protein-protein interactions were found associated with EGF-like 
1 domain (UniProt keyword enrichment, 13 out of 209 genes, FDR=4.2e-04; and INTERPRO

2 protein domain enrichment, 13 out of 191 genes, FDR=2.6e-04). The EGF-like domain contains

3 repeats which bind to apoptotic cells and play a key role in their clearance ${ }^{46}$. Our selective

4 sweep results are consistent with those from the AR analysis and suggest ongoing selection in

5 pathways involved with somatic maintenance and in particular apoptosis, a possible key

6 mechanism for cancer suppression in elephants. 
Figure 5. Selective sweeps in three living elephant species. (a) TreeMap from REVIGO representing semantic clustering of gene ontology biological process terms with a BenjaminiHochberg false discovery rate of $5 \%$ that are associated with genes overlapping selective sweeps common to Elephas maximus, Loxodonta africana, and L. cyclotis. Rectangles represent clusters, and larger rectangles indicate semantically related clusters. Larger rectangle sizes reflect smaller corrected p-values from the GO term enrichment. (b) Composite likelihood ratio values in the NFIC region of a genomic scaffold (loxAfr3.0) calculated with SweeD in three elephant species. Gene annotations are represented by dark rectangles; the NFIC gene is indicated. Dashed lines represent $p$-value threshold of 0.0001 .

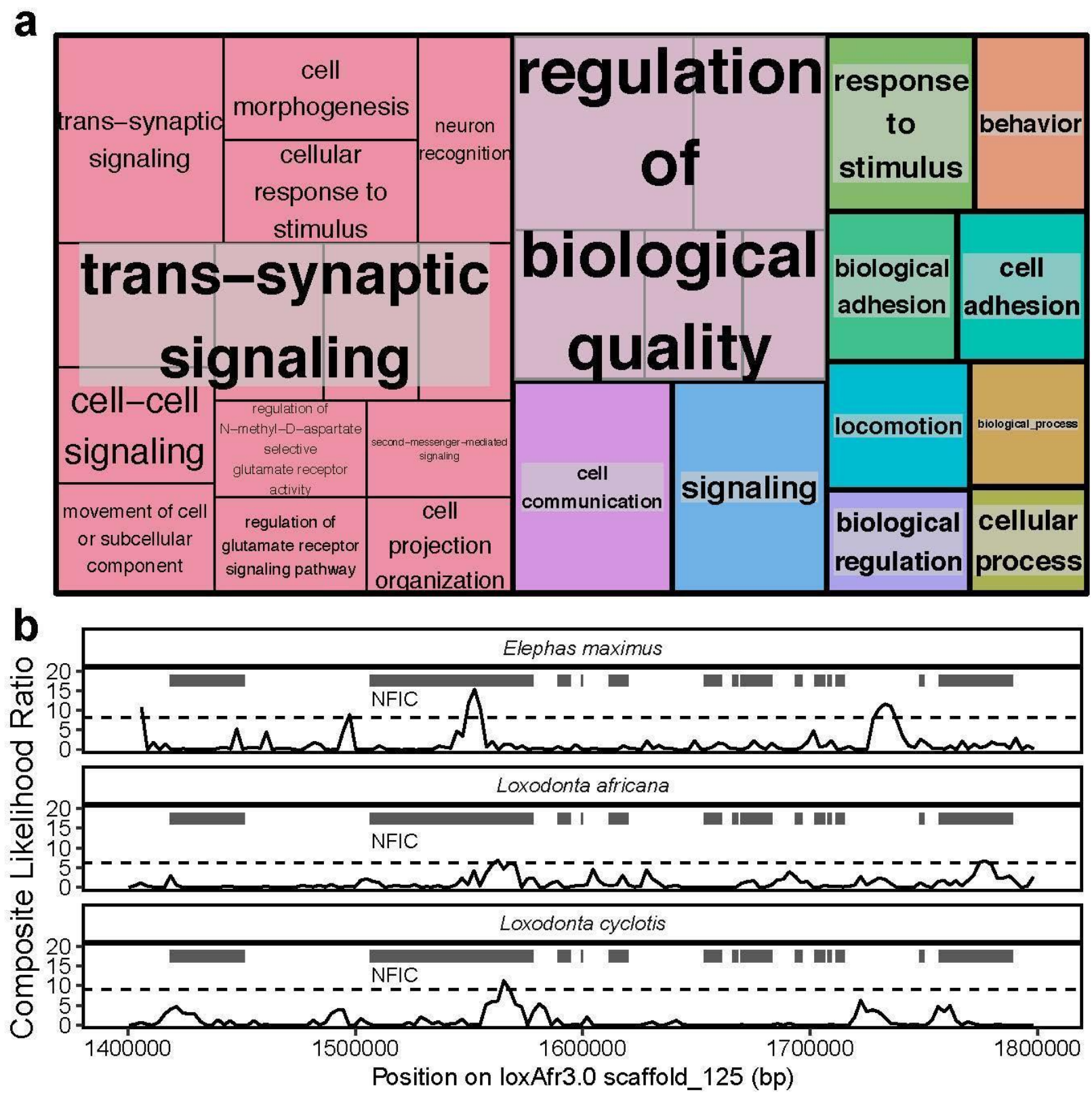




\section{Discussion}

2 Our study of elephant genomes expands the knowledge of elephant evolution, highlighting

3 differences and similarities between species. Elephant tumors tend to be benign with strong

4 genetic defenses to prevent malignant transformation. Asian elephants reported in our study

5 develop benign tumors and malignant cancer at higher rates than African elephants, and

6 therefore may benefit from increased monitoring for tumors. Even though our data originates

7 from captive elephants, these differences most likely reflect true genetic differences as the AZA

8 has a Species Survival Plan (SSP) (https://www.aza.org/species-survival-plan-programs) for

9 elephants that maximizes genetic diversity via the careful selection of mate pairs and studbook

10 documentation ${ }^{47,48}$. Together with the fact that many elephants in zoos are wild born, it is likely

11 that wild Asian elephants share increased susceptibility to neoplasia with the same observed genetic variation we report in our study.

While collecting cancer prevalence data in wild elephants is challenging due to differences in cancer risk between elephant species. This information could benefit the survival of individual elephants and assist with selecting the best treatment interventions when the rare elephant tumor is diagnosed in captivity or in the wild. More than half of the elephant tumors reported here were found in reproductive organs (Table 1). Even benign reproductive tumors can affect reproduction and conservation, therefore future studies to understand their impact

21 and to develop preventative and treatment measures are needed.

While previous studies suggest that TP53 copy number increased with body mass

23 during proboscidean evolution as a response to increased cancer risk ${ }^{14}$, we estimated some of

24 the highest TP53 copy numbers in the smallest elephants. Based on available sequence data,

25 we estimated $\sim 19-21$ TP53 copy number variants in the $\sim 44,800$ year old woolly mammoth

26 genome from Oimyakon, Russia, but found that the much more recent $\sim 4,300$-year-old Wrangel 
1 Island mammoth had $\sim 1.3 \mathrm{X}$ this number of TP53 copies in its genome. These findings are

2 consistent with the demographic decline of the last woolly mammoths on Wrangel Island ${ }^{18}$,

3 which by 12,000 years before present shrunk in body size by $\sim 30 \%$ relative to more ancient

4 mammoths elsewhere ${ }^{4}$. The estimated TP53 copy number increase in the Wrangel Island

5 mammoth may be related to the random fixation of retrogenes in the population rather than

6 selection acting on body size.

We estimated 21-24 TP53 copy number variants in forest elephant genomes, greater

8 than our estimates for bush elephants despite the smaller body size of forest elephants.

9 Meanwhile, we estimated $\sim 23-25$ TP53 copy number variants in the genome of the straight-

10 tusked elephant, which at $\sim 13,000 \mathrm{~kg}$ may have been the largest land mammal to have ever

11 lived (Fig. 3b $)^{49}$. Recent genomic evidence suggests that forest elephants are more closely

12 related to straight-tusked elephants than to bush elephants (Fig. $3 b)^{25,50}$, with extensive gene

13 flow occurring between forest and straight-tusked elephants, as well as between straight-tusked

14 elephants and mammoths ${ }^{25}$. Thus, the possibility exists that, as in the Wrangel Island

15 mammoth, the higher estimated TP53 copy number in forest elephants relative to bush

16 elephants may not be related to modern differences in body mass between species (and

17 possible protection from increased cancer risk), but instead may be due to complicated

18 evolutionary and demographic histories which include migration that can dramatically affect the

19 dynamics of repetitive genomic elements such as retrogenes ${ }^{51}$. Nevertheless, we still find that

20 genetic variation at some TP53 retrogenes is tightly conserved in populations of all living

21 elephant species. This adds at least some evidence to the functionality of TP53 retrogenes. We

22 suggest that there may be a core set of TP53 retrogenes that confer the bulk of cancer

23 suppression in elephants.

24 Our results support the idea that regulatory elements play a role in the increased

25 infectious susceptibility with inflammatory response of Asian versus African elephants,

26 particularly the mediation of the TNF cytokine. Asian elephant calves are much more 
1 susceptible than African elephant calves to cytokine storm triggered by EEHV infection ${ }^{52}$.

2 Compared to African elephants, we found that Asian elephant ARs are enriched for BP GO

3 terms "interleukin-1 beta (IL-1 $\beta$ ) production" (q-value=0.036), "interleukin-18 (IL-18) production"

4 (q-value=0.00073), and "neutrophil activation involved in immune response" (q-value=2.44e-05)

5 (Supplementary Data). IL-1 $\beta$, IL-18 and neutrophils, combined with TNF-alpha, are potent

6 mediators of innate immunity. Uncontrolled activation of these factors leads to immune-induced

7 disease pathogenesis through excessive inflammation. In humans and other mammals,

8 neutrophil activation directly contributes to tissue damage through the release of neutrophil

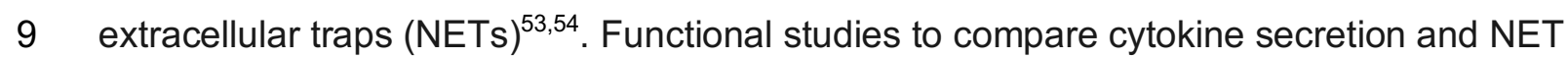

10 release in response to infectious agents are ongoing and could confirm that genetic differences

11 in innate immunity contribute to differences in disease susceptibility and outcomes between

12 Asian and African elephants. Our study provides an example of how genomics can inform

13 functional immunological and molecular studies, which may lead to improved conservation and

14 medical care for elephants. This type of genetic information could provide important evolutionary

15 insights to one day be translated into human patients with infection or cancer.

\section{Materials and Methods}

\section{Cancer Data Collection}

19 Pathology and necropsy records were collected with consent from 26 zoological institutions

20 across the United States over the span of 26 years. All participating institutions were de-

21 identified and anonymized. Data was collected with permissions from Buffalo Zoo, Dallas Zoo,

22 El Paso Zoo, Fort Worth Zoo, Gladys Porter Zoo, Greenville Zoo, Jacksonville Zoo and

23 Gardens, Louisville Zoological Garden, Oakland Zoo, Oklahoma City Zoo and Botanical

24 Garden, Omaha's Henry Doorly Zoo and Aquarium, The Phoenix Zoo, Point Defiance Zoo and

25 Aquarium, San Antonio Zoological Society, Santa Barbara Zoological Gardens, Sedgwick 
1 County Zoo, Seneca Park Zoo, Toledo Zoological Gardens, Utah's Hogle Zoo, Woodland Park

2 Zoo, Zoo Atlanta, Zoo Miami and three other anonymous zoos. Neoplasia was diagnosed by

3 board certified veterinary pathologists and cancers were identified from pathological services at

4 Northwest ZooPath, Monroe, WA. Published pathology data from San Diego Zoo was also

$5 \quad$ included $^{55}$. Neoplasia prevalence was estimated by the number of elephants that were

6 diagnosed with tumors (benign or malignant) in respect to all elephants documented within our

7 database.

8 De novo assembly and annotation of the Asian elephant genome

9 A whole blood sample was drawn in an EDTA tube from the Asian elephant ("Icky", North

10 American studbook number 199) from the Ringling Bros. Center for Elephant Conservation, and

11 DNA libraries were constructed and sequenced at the University of Utah Genomics Core

12 Facility. Paired-end DNA libraries were constructed with the TruSeq Library Prep kit for a target

13 insert size of $200 \mathrm{bp}$, and mate-paired libraries were constructed for target sizes of $3 \mathrm{~kb}, 5 \mathrm{~kb}, 8$

$14 \mathrm{~kb}$, and $10 \mathrm{~kb}$ using the Nextera Mate Pair Library kit. Genomic DNA was sequenced on an

15 Illumina HiSeq2500. Raw reads were trimmed to remove nucleotide biases, adapters and a

16 quality score cutoff of 30 with Trimmomatic $v 0.33^{56}$ and SeqClean ${ }^{57}$. Genome assembly was

17 carried out using ALLPATHS-LG ${ }^{58,59}$. The expected gene content was assessed by searching

18 for 4,104 mammalian single-copy orthologs (mammalia_odb9) using BUSCO v3.0.2 ${ }^{60}$. We

19 annotated and masked repeats in the resulting assembly using both the de novo method

20 implemented in RepeatModeler v1.0.11 ${ }^{61}$ and a database method using RepeatMasker v4.07 ${ }^{62}$

21 with a library of known mammalian repeats from RepBase ${ }^{63}$. Modeled repeats were used in a

22 BLAST search against Swiss-Prot ${ }^{64}$ to identify and remove false positives. We then generated

23 gene models for the Asian elephant assembly using MAKER2 ${ }^{65}$, which incorporated (1)

24 homology to publicly available proteomes of cow, human, mouse, and all mammalian entries in

25 UniProtKB/Swiss-Prot, and (2) ab initio gene predictions based on species-specific gene models 
1 in SNAP $(11 / 29 / 2013 \text { release })^{66}$, species-specific and human gene models in Augustus v3.0.2 ${ }^{67}$,

2 and EvidenceModeler ${ }^{68}$. Final gene calls were functionally annotated by using InterProScan ${ }^{69}$ to

3 identify protein domains and a blastp search of all annotated proteins to UniProt proteins to

4 assign putative orthologs with an e-value cutoff of $1 \mathrm{e}-6$.

5 Tissue collection, DNA extraction, and genome sequencing of African bush elephants

6 The African bush elephant assembly was improved with the addition of $\mathrm{Hi}-\mathrm{C}$ sequencing

7 libraries. First, a whole blood sample was drawn (in an EDTA tube) from a wild-born female

8 named Swazi (animal ID: KB13542, North American studbook number 532) at the San Diego

9 Zoo Safari Park in Escondido, CA. We selected this individual because her genome was

10 originally sequenced by the Broad Institute ${ }^{25}$. Three Hi-C libraries were constructed and

11 sequenced to $\sim 38 \mathrm{X}$ genome coverage and used for scaffolding with HiRise ${ }^{70}$ at Dovetail

12 Genomics in Santa Cruz, CA, with the most recent version of the African bush elephant

13 assembly (loxAfr4.0) as an input. DNA was extracted from fresh frozen subcutaneous skin

14 necropsy tissue samples from an African bush elephant named Hi-Dari (animal ID 00003, North

15 American studbook number 33) at the Hogle Zoo in Salt Lake City, UT using a ThermoFisher

16 PureLink Genomic Mini DNA Kit at the University of Utah. Two pieces of tissue were digested

17 and extracted separately and pooled followed extraction. DNA concentration was measured by

18 PicoGreen (8.66ng/ul) with a total volume of $200 \mathrm{ul}$ in $10 \mathrm{mM} \mathrm{pH} 8.0$. DNA sequencing libraries

19 were generated using the Illumina TruSeq Library Prep Kit for a 350 bp mean insert size, and

20 sequenced on two lanes the Illumina HiSeq2500 platform at Huntsman Cancer Institute's High-

21 Throughput Genomics Core (Salt Lake City, UT).

\section{TP53 evolution in African and Asian elephants}

23 To determine TP53 copy numbers and evolutionary patterns across placental mammals, we

24 exported the TP53 human peptide from Ensembl (July 2019), and used it as a query in

25 reciprocal BLAT searches ${ }^{71}$ of 44 mammalian genome assemblies (Supplementary Table 6), 
validated with a BLASTX query of the human peptide database on NCBI in order to ensure the

top hit was human TP53 with $\geq 70 \%$ protein identity, following Tollis et al. $(2020)^{72}$. Accepted

3 nucleotide sequences were aligned with $\mathrm{MAFFT}^{73}$, and we weighted and filtered out unreliable

4 columns in the alignment with GUIDANCE2 ${ }^{74}$ using 100 bootstrap replicates. We reconstructed

5 the phylogeny of all aligned mammalian TP53 homologs and estimated their divergence times in

6 a Bayesian framework with BEAST $2.5^{75}$ using the HKY substitution model, a relaxed lognormal

7 molecular clock model, and a Yule Model tree prior. We used a normal prior distribution for the

8 age of Eutheria (offset to 105 million years or MYA with the $2.5 \%$ quantile at 101 MYA and the

$997.5 \%$ quantile at 109 MYA) and lognormal prior distributions for the following node calibrations

10 from the fossil record ${ }^{61}$ : Boreoeutheria (offset the minimum age to 61.6 MYA -164 MYA and the

$1197.5 \%$ quantile to 170 MYA), Euarchontoglires (56 MYA - 164 MYA), Primates (56 MYA - 66

12 MYA), Laurasiatheria (61.6 MYA - 164 MYA) and Afrotheria (56 MYA - 164 MYA). We

13 monitored proper MCMC mixing with Tracer v1.7.1 ${ }^{76}$ to ensure an effective sampling size of at

14 least 200 from the posterior distributions of each parameter and ran two independent chains.

15 The final MCMC chain was run for 100,000,000 generations, and we logged parameter samples

16 every 10,000 generations to collect a total of 10,000 samples from the posterior distribution. We

17 then collected 10,000 of the resulting trees, ignored the first $10 \%$ as burn-in, and calculated the

18 maximum clade credibility tree using TreeAnnotator.

\section{Detection of accelerated regions in African and Asian elephant genomes}

20 We generated a multiple alignment (whole genome alignment or WGA) of 12 mammalian

21 genome assemblies. First, we downloaded publicly available pairwise syntenic alignments of

22 opossum (monDom5), mouse (mm10), dolphin (turTru1), cow (bosTau7), dog (canFam3), horse

23 (equCab2), microbat (myoLuc1), tenrec (echTel2), and hyrax (proCap1) to the human reference

24 (hg19) from the UCSC Genome Browser ${ }^{77}$. We also computed two additional de novo pairwise 
1 syntenic alignments with the human genome as a target and the two elephant genome

2 assemblies reported here as queries using local alignments from LASTZ v1.02 ${ }^{78}$ using the

3 following options from the UCSC Genome Browser for mammalian genome alignments: --

4 hspthresh 2200 --inner 2000 --ydrop 3400 --gappedthresh 10000 --scores HOXD70, followed by

5 chaining to form gapless blocks and netting to rank the highest scoring chains ${ }^{79}$. We then

6 constructed a multiple sequence alignment with MULTIZ v11.2 ${ }^{80}$ with human as the reference

7 species.

8 To define elephant accelerated regions (ARs), we used functions from the R package

9 rphast $\mathrm{v} 1.6^{24}$. We used phyloFit with the substitution model 'REV' to estimate a neutral model

10 based on autosomal fourfold degenerate sites from the WGA. We then used phastCons to

11 define $60 \mathrm{bp}$ autosomal regions conserved in the 10 non-elephant species in the WGA with the

12 following options: expected.length $=45$, target.coverage $=0.3$, rho $=0.31$. We further selected

13 regions with aligned sequence for both African and Asian elephants that have aligned sequence

14 present for at least 9 of the 10 non-elephant species. We tested the resulting 676,509 regions

15 for acceleration in each elephant species relative to the 10 non-elephant species with phyloP

16 using the following options: mode = 'ACC'. We used the Q-Value method ${ }^{81}$ to adjust for multiple

17 testing. Statistically significant ARs were defined with a false discovery rate threshold of $10 \%$.

18 We defined regions significantly accelerated in the Asian elephant, but not the African bush

19 elephant as Asian elephant specific ARs and conversely defined African bush elephant specific

20 ARs. Our previous studies of accelerated regions suggest no significant relationship between

21 genome quality and number of ARs discovered ${ }^{19}$.

To define genes differentially expressed between Asian and African elephants we took

23 advantage of the closeness between the two species. The Asian elephant is more closely

24 related to the African elephant than humans are to chimpanzees (0.01186 substitutions / $100 \mathrm{bp}$

25 vs 0.01277 substitutions / 100 bp based on fourfold degenerate sites from our WGA). For the

26 purpose of defining differentially expressed genes, chimpanzee RNA-Seq reads have been 
1 aligned to human transcriptome indices ${ }^{82}$. We aligned African bush elephant PBMC reads (four

2 technical replicates) from a previous study ${ }^{19}$ and publicly available Asian elephant PBMC data

3 from a single individual ${ }^{28}$ (one replicate) to an African elephant (loxAfr3) transcriptome index

4 with the STAR aligner ${ }^{83}$. After counting reads for each elephant gene with featureCounts ${ }^{84}$, we

5 normalized counts with the TMM method and defined significant DE genes with edgeR ${ }^{85}$ (FDR <

6 0.01) correcting for multiple testing with the Benjamini-Hochberg method. The DE gene list was

7 minimally affected by modest FDR cutoff changes. We note differences in the cell preps, RNA

8 purification methods and sex of the Asian and African elephants as potential confounding

9 factors in defining DE genes. The African elephant PBMC RNA was purified with a Ribo-Zero

10 depletion step while the Asian elephant RNA was purified by Poly-A selection. A study

11 comparing the two RNA purification methods shows a high gene expression correlation (0.931)

12 between the two methods and detects 410 genes as differentially expressed when contrasting

13 these purification methods ${ }^{86}$.

Potential regulatory regions for elephant DE genes were defined with custom $\mathrm{R}$ scripts

15 implementing logic detailed by McLean et al. (2010) ${ }^{87}$ based on transcription start site (TSS)

16 locations obtained for protein coding genes with the R package biomaRt ${ }^{88}$ for the African bush

17 elephant genome (loxAfr3) with basal distances of $5 \mathrm{~kb}$ upstream and $1 \mathrm{~kb}$ downstream an

18 extension distance of $100 \mathrm{~kb}$. We chose this extension distance because the majority of

19 conserved enhancers are located within $100 \mathrm{~kb}$ of a TSS ${ }^{89}$. We used the R package LOLA ${ }^{90}$ to

20 test for enrichment of ARs relative to CRs in the potential regulatory regions of DE genes in the

21 loxAfr3 genome. Biological processes (BP) and associated elephant orthologs of human genes

22 were obtained with biomaRt. The resulting p-values were q-value corrected for multiple

23 testing ${ }^{81}$. We used the same potential regulatory regions and LOLA to test for GO enrichments.

24 We compared elephant AR set GO enrichments to GO enrichments from previously

25 published AR sets for 5 mammalian species (13-lined ground squirrel, naked mole rat, orca,

26 bottlenose dolphin, and little brown bat $)^{19}$. These AR sets were lifted over from hg19 coordinates 
1 to loxAfr3 coordinates. Numbers of ARs and background CRs overlapping potential regulatory

2 regions of genes included in and excluded from each GO term were calculated with LOLA. We

3 used generalized linear models with binomial distributions to compare elephant AR enrichments

4 in each GO term to AR enrichments for the 5 other mammals. We contrasted models without

5 and with an interaction term distinguishing the elephant AR set from the others. The two models

6 are

7

$\log \left[\frac{P(\mathrm{ar})}{1-P(\mathrm{ar})}\right]=\alpha+\beta_{1}\left(\mathrm{~g}_{\mathrm{t}}\right)+\beta_{2}\left(\mathrm{c}_{\mathrm{e}}\right)+\epsilon$

$\log \left[\frac{P(\mathrm{ar})}{1-P(\mathrm{ar})}\right]=\alpha+\beta_{1}\left(\mathrm{~g}_{\mathrm{t}}\right)+\beta_{2}\left(\mathrm{c}_{\mathrm{e}}\right)+\beta_{3}\left(\mathrm{~g}_{\mathrm{t}} \times \mathrm{c}_{\mathrm{e}}\right)+\epsilon$

11 where $g_{t}$ is a binary value $\{0,1\}$ indicating gene regions excluded from or included in a given $\mathrm{GO}$

12 term set; $c_{e}$ is a binary value $\{0,1\}$ indicating non-elephant or elephant accelerated regions

13 study; ar is the number of ARs in a given category. For each GO term with significant AR

14 enrichments for at least one of the three elephant AR sets in the earlier analysis, we determined

15 the significance of the enrichment in each elephant AR set relative to the other mammal AR sets

16 by comparing the two models by likelihood ratio test. The likelihood ratio test p-values are

17 reported in the Supplementary Data.

Whole genome sequence analysis of living elephants

19 We obtained $\sim 15-40 x$ whole-genome sequencing data from multiple individuals from across the

20 modern range of living elephants from public databases ${ }^{12,16,28,91}$, and the WGS libraries for "Hi-

21 Dari" and "Icky" as well as a third African elephant named "Christie" (Supplementary Table 7).

22 We also obtained sequence data from a straight-tusked elephant ${ }^{25}$ and two woolly mammoths ${ }^{91}$.

23 Sequences were quality checked using FastQC and trimmed to remove nucleotide biases and

24 adapter sequences with Trimmomatic where necessary. Reads from each individual were 
1 mapped to the $L$. africana genome (loxAfr3.0, Ensembl version) using bwa-mem v07792.

2 Alignments were filtered to include only mapped reads and sorted by position using Samtools

3 v0.0.1993, and we removed PCR duplicates using MarkDuplicates in picard v1.125 $5^{94}$. Single-end

4 reads from the ancient samples were mapped to loxAfr3.0 with bwa-aln following Palkolpoulou

5 et al. (2018).

We estimated the number of TP53 paralogs present in the genome of each elephant by calculating the average read depth of annotated sites in Ensembl TP53 exons and whole genes with Samtools, dividing the total average genome coverage, multiplied by the number of TP53 annotations $(n=12)$. We called variants in the living elephant species $(n=13)$ by incorporating the .bam files using freebayes $v 1 \cdot 3 \cdot 1-12^{36}$, with extensive filtering to avoid false positives as follows with vcffilter from vcflib (https://github.com/vcflib/vcflib, last accessed July 2019): Phred-scale probability that a REF/ALT polymorphism exists at a given site (QUAL) > 20, the additional contribution of each observation should be 10 log units (QUAL/AO>10), read depth (DP>5), reads must exist on both strands $(S A F>0 \& S A R>0)$, and at least two reads must be balanced to each side of the site (RPR>1 \& RPL $>1)$. We then removed indels from the .vcf file and filtered to only include biallelic SNPs that were genotyped in every individual using VCFtools v0.1.17 ${ }^{95}$ (-remove-indels --min-alleles 2 --max-alleles 2 --max-missing-count 0) and bcftools v1.996 (-v snps $-\mathrm{m} 1$ ). We annotated the biallelic SNPs using SnpEff v4.3 ${ }^{97}$ based on loxAfr3 (Ensembl), and calculated diversity statistics including per-individual heterozygosity, nucleotide diversity

20 and Tajima's $D$ in $10 \mathrm{~kb}$ windows with VCFtools, and the fixation index $F_{S T}$ with PopGenome

21 v2.7.198. We estimated RoH with PLINK v1.999 using the following parameter settings: --

22 homozyg-window-snp 100 --homozyg-window-missing 15 --homozyg-window-het 5 --homozyg-

23 window-threshold 0.05 --homozyg-snp 25 --homozyg-kb 100 --homozyg-density 50 --homozyg-

24 gap 1000 --homozyg-het 750 --allow-extra-chr. For each elephant, the inbreeding coefficient

$25\left(F_{R O H}\right)^{100}$ was estimated using the total length of $\mathrm{RoH} \geq 500 \mathrm{~Kb}$. 


\section{Selective sweep analysis}

2 To detect loci that have been putatively subjected to positive selection within each living

3 elephant species, we used SweeD v3.3.1. SweeD scans the genome for selective sweeps by

4 calculating the composite likelihood ratio (CLR) test from the folded site frequency spectrum in 1

$5 \mathrm{~kb}$ grids across each scaffold. We used the folded site frequency spectrum because we lacked

6 a suitable closely related outgroup with genomic resources that would enable us to establish

7 ancestral alleles. For this analysis, we studied each species individually. Following Nielsen et al.

8 (2005), we established statistical thresholds for this outlier analysis. First, we generated a null

9 model by simulating 1,000 data sets with 100,000 SNPs under neutral demographic models. As

10 our population genomics statistics results were highly concordant with Palkopoulou et al. (2018),

11 we constructed demographic models based on their Pairwise Sequential Markovian Coalescent

12 trajectories ${ }^{101}$ for each species, which we implemented with ms (October 2007 release) ${ }^{102}$

13 (Supplementary Fig. 9). Then, we categorized regions as outlier regions in the observed SNP

14 data if their CLR was greater than the $99.99^{\text {th }}$ percentile of the distribution of the highest CLRs

15 from the simulated SNP data. For the neutral simulations, we assumed a per-year mutation rate

16 of $0.406 e-09$ and a generation time of 31 years, following Palkopoulou et al. (2018). We then

17 calculated the CLR with the simulated neutral SNP datasets. SweeD output files were changed

18 to BED format using namedCapture ${ }^{103}$ and data.table ${ }^{104} \mathrm{R}$ packages, and we used bedtools

19 intersect ${ }^{105}$ to collect elephant gene annotations (loxAfr3.0, Ensembl) overlapping putative

20 selective sweeps.

21 Genomic scans for selection may be complicated by several factors that can increase

22 false positive rates, and false negative rates potentially stem from variable mutation and

23 recombination landscapes ${ }^{38,106}$. We established statistical thresholds using null demographic

24 models. However, the estimated split times within living elephant species differ widely, ranging

25 from 609,000 to 463,000 years before present for forest elephants ${ }^{37}$, to as recent as 38,000 to

2630,000 years before present for bush elephants ${ }^{91,107}$. Estimated split times between the sampled 
1 Asian elephants are highly variable, ranging from 190,000 to 24,000 years before present ${ }^{25}$,

2 indicating continental-wide population structure not accounted for here ${ }^{108,109}$. Still, Palkopoulou

3 et al. (2018) found little evidence for gene flow between the three modern species of elephant,

4 which supports our choice of analyzing them separately for selective sweeps. We focused on

5 shared outlier regions, which show consistent evidence of being targeted by positive selection

6 across all three elephant species.

$7 \quad$ Genes overlapping outlier regions of putative selective sweeps were functionally

8 annotated by testing for GO enrichment of terms for biological processes ${ }^{110}$ in the outlier gene

9 list, using default parameters and the Benjamini-Hochberg correction for multiple testing with an

10 adjusted $p$-value $<0.05$. We used REVIGO ${ }^{111}$ to semantically cluster and visualize the most

11 significant GO terms according to their adjusted p-values using default parameters. We also

12 created annotation clusters from the outlier genes using DAVID v6.8 $8^{112}$ and constructed protein

13 interaction networks with STRING v11.0 ${ }^{113}$. Finally, we tested for enriched mouse phenotypes

14 using ModPhea ${ }^{114}$.

15 Data Availability

16 Short-read sequence data generated for this study has been shared under NCBI Bioproject

17 PRJNA622303, and the genome assembly for Icky the Asian elephant is available on NCBI

18 (GCA_014332765.1). Other datasets including the updated African elephant genome assembly,

19 annotation files for Asian elephant, multiple genome alignments, TP53 alignments and

20 phylogeny, .vcf files, and selective sweep results have been deposited to Zenodo

21 (https://zenodo.org/record/4033444\#.X5clSFNKhGp).

\section{Acknowledgements}

23 We acknowledge Leigh Duke for data coordination and Trent Fowler and Rosann Robinson for

24 assistance with sample collection. We acknowledge the collections and veterinary staff at the

25 San Diego Zoo Safari Park and Utah's Hogle Zoo for sample collection. We acknowledge the 
following institutions for sharing data and/or resources: Buffalo Zoo, Dallas Zoo, El Paso Zoo,

2 Fort Worth Zoo, Gladys Porter Zoo, Greenville Zoo, Jacksonville Zoo and Gardens, Louisville

3 Zoological Garden, Oakland Zoo, Oklahoma City Zoo and Botanical Garden, Omaha's Henry

4 Doorly Zoo and Aquarium, The Phoenix Zoo, Point Defiance Zoo and Aquarium, San Antonio

5 Zoological Society, Santa Barbara Zoological Gardens, Sedgwick County Zoo, Seneca Park

6 Zoo, Toledo Zoological Gardens, Utah's Hogle Zoo, Woodland Park Zoo, Zoo Atlanta, Zoo

7 Miami and three other anonymous zoos. We acknowledge Huntsman Cancer Institute's High-

8 Throughput Genomics Core and the Monsoon computing cluster at Northern Arizona University

9 (https://nau.edu/high-performance-computing/). Research reported in this publication was

10 supported by the National Cancer Institute of the National Institutes of Health under Award

11 Number U54CA217376. The content is solely the responsibility of the authors and does not

12 necessarily represent the official views of the National Institutes of Health.

\section{Conflict of Interest}

Dr. Schiffman is co-founder, shareholder, and employed by PEEL Therapeutics, Inc., a

company developing evolution-inspired medicines based on cancer resistance in elephants. Dr.

Abegglen is share-holder and consultant to PEEL Therapeutics, Inc.

\section{References}

1. Shoshani, J. Understanding proboscidean evolution: a formidable task. Trends Ecol. Evol. 13, 480-487 (1998).

2. Vartanyan, S. L., Garutt, V. E. \& Sher, A. V. Holocene dwarf mammoths from Wrangel Island in the Siberian Arctic. Nature 362, 337-340 (1993).

3. Stuart, A. J. The extinction of woolly mammoth (Mammuthus primigenius) and straighttusked elephant Palaeoloxodon antiquus) in Europe. Quat. Int. 126-128, 171-177 (2005).

4. Fernando, P. \& Pastorini, J. Range-wide status of Asian elephants. Gajah 35, 15-20 (2011).

5. Blanc, J. J. et al. African elephant status report 2007: an update from the African elephant database. (2007) doi:10.2305/IUCN.CH.2007.SSC-OP.33.en.

6. Thouless, C. R. et al. African elephant status report 2016: an update from the African Elephant Database. Occas. Pap. IUCN Surviv. Comm. 60, (2016).

7. Chapman, S. N., Jackson, J., Htut, W., Lummaa, V. \& Lahdenperä, M. Asian elephants exhibit post-reproductive lifespans. BMC Evol. Biol. 19, 193 (2019).

8. Moss, C. J. The demography of an African elephant (Loxodonta africana) population in Amboseli, Kenya. J. Zool. 255, 145-156 (2001). 
9. Greenwald, R. et al. Highly accurate antibody assays for early and rapid detection of tuberculosis in African and Asian elephants. Clin. Vaccine Immunol. 16, 605-612 (2009).

10. Long, S. Y., Latimer, E. M. \& Hayward, G. S. Review of elephant endotheliotropic herpesviruses and acute hemorrhagic disease. ILAR J. 56, 283-296 (2016).

11. Srivorakul, S. et al. Possible roles of monocytes/macrophages in response to elephant endotheliotropic herpesvirus (EEHV) infections in Asian elephants (Elephas maximus). PLOS ONE 14, e0222158 (2019).

12. Abegglen, L. M. et al. Potential mechanisms for cancer resistance in elephants and comparative cellular response to DNA damage in humans. JAMA 314, 1850-1860 (2015).

13. Caulin, A. F., Graham, T. A., Wang, L.-S. \& Maley, C. C. Solutions to Peto's paradox revealed by mathematical modelling and cross-species cancer gene analysis. Philos. Trans. R. Soc. Lond. B. Biol. Sci. 370, 20140222 (2015).

14. Sulak, M. et al. TP53 copy number expansion is associated with the evolution of increased body size and an enhanced DNA damage response in elephants. eLife 5, 1850 (2016).

15. Supple, M. A. \& Shapiro, B. Conservation of biodiversity in the genomics era. Genome Biol. 19, 131 (2018).

16. Lynch, V. J. et al. Elephantid genomes reveal the molecular bases of woolly mammoth adaptations to the Arctic. Cell Rep. 1-13 (2015) doi:10.1016/j.celrep.2015.06.027.

17. Fry, E. et al. Functional architecture of deleterious genetic variants in the genome of a Wrangel Island mammoth. Genome Biol. Evol. 12, 48-58 (2020).

18. Rogers, R. L. \& Slatkin, M. Excess of genomic defects in a woolly mammoth on Wrangel island. PLoS Genet. 13, e1006601 (2017).

19. Ferris, E., Abegglen, L. M., Schiffman, J. D. \& Gregg, C. Accelerated evolution in distinctive species reveals candidate elements for clinically relevant traits, including mutation and cancer resistance. Cell Rep. 22, 2742-2755 (2018).

20. Howlander, N. et al. SEER Cancer Statistics Review, 1975-2017. https://seer.cancer.gov/csr/1975_2017/ (2020).

21. Zimmermann, A., Bernuit, D., Gerlinger, C., Schaefers, M. \& Geppert, K. Prevalence, symptoms and management of uterine fibroids: an international internet-based survey of 21,746 women. BMC Womens Health 12, 6 (2012).

22. Montali, R. J. et al. Ultrasonography and pathology of genital tract leiomymoas in captive asian elephants: implications for reproductive soundness. Erkrangungen Zootiere 38, (1997).

23. Siepel, A. et al. Evolutionarily conserved elements in vertebrate, insect, worm, and yeast genomes. Genome Res. 15, 1034-1050 (2005).

24. Hubisz, M. J., Pollard, K. S. \& Siepel, A. PHAST and RPHAST: phylogenetic analysis with space/time models. Brief. Bioinform. 12, 41-51 (2011).

25. Palkopoulou, E. et al. A comprehensive genomic history of extinct and living elephants. Proc. Natl. Acad. Sci. U. S. A. 115, E2566-E2574 (2018).

26. Pollard, K. S., Hubisz, M. J., Rosenbloom, K. R. \& Siepel, A. Detection of nonneutral substitution rates on mammalian phylogenies. Genome Res. 20, 110-121 (2010).

27. Booker, B. M. et al. Bat Accelerated Regions Identify a Bat Forelimb Specific Enhancer in the HoxD Locus. PLOS Genet. 12, e1005738 (2016).

28. Reddy, P. C. et al. Comparative sequence analyses of genome and transcriptome reveal novel transcripts and variants in the Asian elephant Elephas maximus. J. Biosci. 40, 891907 (2015).

29. Hetz, C. A. et al. Caspase-dependent initiation of apoptosis and necrosis by the Fas receptor in lymphoid cells: onset of necrosis is associated with delayed ceramide increase. J. Cell Sci. 115, 4671-4683 (2002).

30. Venable, M. E., Lee, J. Y., Smyth, M. J., Bielawska, A. \& Obeid, L. M. Role of ceramide in cellular senescence. J. Biol. Chem. 270, 30701-30708 (1995). 
31. Snider, A. J., Orr Gandy, K. A. \& Obeid, L. M. Sphingosine kinase: Role in regulation of bioactive sphingolipid mediators in inflammation. Biochimie 92, 707-715 (2010).

32. Moldovan, G.-L. \& D'Andrea, A. D. How the Fanconi anemia pathway guards the genome. Annu. Rev. Genet. 43, 223-249 (2009).

33. Clavijo, B. J. et al. W2RAP: a pipeline for high quality, robust assemblies of large complex genomes from short read data. bioRxiv 110999 (2017) doi:10.1101/110999.

34. Dudchenko, O. et al. De novo assembly of the Aedes aegypti genome using Hi-C yields chromosome-length scaffolds. Science 356, 92-95 (2017).

35. Dudchenko, O. et al. The Juicebox Assembly Tools module facilitates de novo assembly of mammalian genomes with chromosome-length scaffolds for under \$1000. bioRxiv 254797 (2018) doi:10.1101/254797.

36. Garrison, E. \& Marth, G. Haplotype-based variant detection from short-read sequencing. (2012).

37. Rohland, N. et al. Genomic DNA sequences from mastodon and woolly mammoth reveal deep speciation of forest and savanna elephants. PLoS Biol. 8, e1000564 (2010).

38. Nielsen, R. et al. Genomic scans for selective sweeps using SNP data. Genome Res. 15, 1566-1575 (2005).

39. Pavlidis, P., Živkovic, D., Stamatakis, A. \& Alachiotis, N. SweeD: likelihood-based detection of selective sweeps in thousands of genomes. Mol. Biol. Evol. 30, 2224-2234 (2013).

40. Zhou, Y. \& Danbolt, N. C. Glutamate as a neurotransmitter in the healthy brain. J. Neural Transm. 121, 799-817 (2014).

41. Haug, $\mathrm{H}$. Comparative studies of the brains of men, elephants and toothed whales. Verh. Anat. Ges. 64, 191-195 (1970).

42. Stránecký, V. et al. Mutations in ANTXR1 cause GAPO syndrome. Am. J. Hum. Genet. 92 , 792-799 (2013).

43. Dinckan, N. et al. A biallelic ANTXR1 variant expands the Anthrax Toxin Receptor Associated phenotype to tooth agenesis. Am. J. Med. Genet. A. 176, 1015-1022 (2018).

44. Jiang, Q. et al. Antxr1, Which is a Target of Runx2, Regulates Chondrocyte Proliferation and Apoptosis. Int. J. Mol. Sci. 21, 2425 (2020).

45. Kim, T.-H., Bae, C.-H., Yang, S., Park, J.-C. \& Cho, E.-S. Nfic regulates tooth root patterning and growth. Anat. Cell Biol. 48, 188-194 (2015).

46. Park, S.-Y., Kim, S.-Y., Jung, M.-Y., Bae, D.-J. \& Kim, I.-S. Epidermal growth factor-like domain repeat of stabilin-2 recognizes phosphatidylserine during cell corpse clearance. Mol. Cell. Biol. 28, 5288-5298 (2008).

47. Lei, R., Brenneman, R. A. \& Louis, E. E. Genetic diversity in the North American captive African elephant collection. J. Zool. 275, 252-267 (2008).

48. Lei, R., Brenneman, R. A., Schmitt, D. L. \& Louis, E. E. Genetic diversity in North American captive Asian elephants. J. Zool. 286, 38-47 (2012).

49. Larramendi, A. Shoulder height, body mass, and shape of proboscideans. Acta Palaeontol. Pol. 61, 537-574 (2015).

50. Meyer, M. et al. Palaeogenomes of Eurasian straight-tusked elephants challenge the current view of elephant evolution. eLife 6, 1175 (2017).

51. Deceliere, G., Charles, S. \& Biémont, C. The dynamics of transposable elements in structured populations. Genetics 169, 467-474 (2005).

52. Hayward, G. S. Conservation: clarifying the risk from herpesvirus to captive Asian elephants. Vet. Rec. 170, 202-203 (2012).

53. Jorch, S. K. \& Kubes, P. An emerging role for neutrophil extracellular traps in noninfectious disease. Nat. Med. 23, 279-287 (2017).

54. Goggs, R., Jeffery, U., LeVine, D. N. \& Li, R. H. L. Neutrophil-extracellular traps, cell-free DNA, and immunothrombosis in companion animals: A review. Vet. Pathol. 57, 6-23 (2020).

55. Boddy, A. M. et al. Lifetime cancer prevalence and life history traits in mammals. Evol. Med. 
Public Health doi:10.1093/emph/eoaa015.

56. Bolger, A. M., Lohse, M. \& Usadel, B. Trimmomatic: a flexible trimmer for lllumina sequence data. Bioinformatics 30, 2114-2120 (2014).

57. Chen, Y.-A., Lin, C.-C., Wang, C.-D., Wu, H.-B. \& Hwang, P.-I. An optimized procedure greatly improves EST vector contamination removal. BMC Genomics 8, 416 (2007).

58. MacCallum, I. et al. ALLPATHS 2: small genomes assembled accurately and with high continuity from short paired reads. Genome Biol. 10, R103 (2009).

59. Gnerre, S. et al. High-quality draft assemblies of mammalian genomes from massively parallel sequence data. Proc. Natl. Acad. Sci. U. S. A. 108, 1513-1518 (2011).

60. Simão, F. A., Waterhouse, R. M., loannidis, P., Kriventseva, E. V. \& Zdobnov, E. M. BUSCO: assessing genome assembly and annotation completeness with single-copy orthologs. Bioinformatics 31, btv351-3212 (2015).

61. Smit, A. F. A., Hubley, R. M. \& Green, P. RepeatModeler Open-1.0 2008-2015. http://www.repeatmasker.org.

62. Smit, A. F. A., Hubley, R. M. \& Green, P. RepeatMasker Open-4.0 2013-2015. httpwww.repeatmasker.org.

63. Jurka, J. et al. Repbase Update, a database of eukaryotic repetitive elements. Cytogenet. Genome Res. 110, 462-467 (2005).

64. UniProt Consortium. UniProt: a hub for protein information. Nucleic Acids Res. 43, D204-12 (2015).

65. Holt, C. \& Yandell, M. MAKER2: an annotation pipeline and genome-database management tool for second-generation genome projects. BMC Bioinformatics 12, 491 (2011).

66. Korf, I. Gene finding in novel genomes. BMC Bioinformatics 5, 59 (2004).

67. Stanke, M., Diekhans, M., Baertsch, R. \& Haussler, D. Using native and syntenically mapped cDNA alignments to improve de novo gene finding. Bioinformatics 24, 637-644 (2008).

68. Haas, B. J. et al. Automated eukaryotic gene structure annotation using EVidenceModeler and the Program to Assemble Spliced Alignments. Genome Biol. 9, R7 (2008).

69. Jones, P. et al. InterProScan 5: genome-scale protein function classification. Bioinformatics 30, 1236-1240 (2014).

70. Putnam, N. H. et al. Chromosome-scale shotgun assembly using an in vitro method for long-range linkage. Genome Res. 26, 342-350 (2016).

71. Kent, W. J. BLAT--the BLAST-like alignment tool. Genome Res. 12, 656-664 (2002).

72. Tollis, M., Schneider-Utaka, A. K. \& Maley, C. C. The evolution of human cancer gene duplications across mammals. Mol. Biol. Evol. 37, 2875-2886 (2020).

73. Katoh, K. \& Standley, D. M. MAFFT multiple sequence alignment software version 7: improvements in performance and usability. Mol. Biol. Evol. 30, 772-780 (2013).

74. Sela, I., Ashkenazy, H., Katoh, K. \& Pupko, T. GUIDANCE2: accurate detection of unreliable alignment regions accounting for the uncertainty of multiple parameters. Nucleic Acids Res. 43, W7-14 (2015).

75. Bouckaert, R. et al. BEAST 2: A Software Platform for Bayesian Evolutionary Analysis. PLOS Comput. Biol. 10, e1003537 (2014).

76. Rambaut, A., Drummond, A. J., Xie, D., Baele, G. \& Suchard, M. A. Posterior summarization in Bayesian phylogenetics using Tracer 1.7. Syst. Biol. 67, 901-904 (2018).

77. Kent, W. J. et al. The human genome browser at UCSC. Genome Res. 12, 996-1006 (2002).

78. Harris, R. S. Improved Pairwise Alignment of Genomic DNA. PhD Thesis (2007).

79. Kent, W. J., Baertsch, R., Hinrichs, A., Miller, W. \& Haussler, D. Evolution's cauldron: duplication, deletion, and rearrangement in the mouse and human genomes. Proc. Natl. Acad. Sci. 100, 11484-11489 (2003).

80. Blanchette, M. et al. Aligning multiple genomic sequences with the threaded blockset 
aligner. Genome Res. 14, 708-715 (2004).

81. Storey, J. D. The positive false discovery rate: a Bayesian interpretation and the q-value. Ann. Stat. 31, 2013-2035 (2003).

82. Marchetto, M. C. et al. Species-specific maturation profiles of human, chimpanzee and bonobo neural cells. eLife 8, e37527 (2019).

83. Dobin, A. et al. STAR: ultrafast universal RNA-seq aligner. Bioinformatics 29, 15-21 (2013).

84. Liao, Y., Smyth, G. K. \& Shi, W. featureCounts: an efficient general purpose program for assigning sequence reads to genomic features. Bioinformatics 30, 923-930 (2014).

85. Robinson, M. D., McCarthy, D. J. \& Smyth, G. K. edgeR: a Bioconductor package for differential expression analysis of digital gene expression data. Bioinformatics 26, 139-140 (2010).

86. Zhao, W. et al. Comparison of RNA-Seq by poly (A) capture, ribosomal RNA depletion, and DNA microarray for expression profiling. BMC Genomics 15, 419 (2014).

87. McLean, C. Y. et al. GREAT improves functional interpretation of cis -regulatory regions. Nat. Biotechnol. 28, 495-501 (2010).

88. Durinck, S. et al. BioMart and Bioconductor: a powerful link between biological databases and microarray data analysis. Bioinformatics 21, 3439-3440 (2005).

89. Villar, D. et al. Enhancer evolution across 20 mammalian species. Cell 160, 554-566 (2015).

90. Sheffield, N. C. \& Bock, C. LOLA: enrichment analysis for genomic region sets and regulatory elements in $\mathrm{R}$ and Bioconductor. Bioinformatics 32, 587-589 (2016).

91. Palkopoulou, E. et al. Complete genomes reveal signatures of demographic and genetic declines in the woolly mammoth. Curr. Biol. CB 25, 1395-1400 (2015).

92. Li, H. \& Durbin, R. Fast and accurate short read alignment with Burrows-Wheeler transform. Bioinformatics 25, 1754-1760 (2009).

93. Li, H. et al. The Sequence Alignment/Map format and SAMtools. Bioinformatics 25, 20782079 (2009).

94. DePristo, M. A. et al. A framework for variation discovery and genotyping using nextgeneration DNA sequencing data. Nat. Genet. 43, 491-498 (2011).

95. Danecek, P. et al. The variant call format and VCFtools. Bioinformatics 27, 2156-2158 (2011).

96. Danecek, P. \& McCarthy, S. A. BCFtools/csq: haplotype-aware variant consequences. Bioinformatics 33, 2037-2039 (2017).

97. Cingolani, P. et al. A program for annotating and predicting the effects of single nucleotide polymorphisms, SnpEff: SNPs in the genome of Drosophila melanogaster strain w1118; iso2; iso-3. Fly (Austin) 6, 80-92 (2012).

98. Pfeifer, B., Wittelsbürger, U., Ramos-Onsins, S. E. \& Lercher, M. J. PopGenome: an efficient Swiss army knife for population genomic analyses in R. Mol. Biol. Evol. 31, 19291936 (2014).

99. Purcell, S. et al. PLINK: A tool set for whole-genome association and population-based linkage analyses. Am. J. Hum. Genet. 81, 559-575 (2007).

100. Forutan, M. et al. Inbreeding and runs of homozygosity before and after genomic selection in North American Holstein cattle. BMC Genomics 19, 98 (2018).

101. Li, H. \& Durbin, R. Inference of human population history from individual whole-genome sequences. Nature 475, 493-496 (2011).

102. Hudson, R. R. Generating samples under a Wright-Fisher neutral model of genetic variation. Bioinformatics 18, 337-338 (2002).

103. Hocking, T., Dylan. Comparing namedCapture with other $\mathrm{R}$ packages for regular expressions. R J. 11, 328 (2019).

104. Dowle, M., Srinivasan, A., Gorecki, J., 'data, M. C. E. of \& 2019. Package 'data. table'. musicbrainz.org. 
105. Quinlan, A. R. \& Hall, I. M. BEDTools: a flexible suite of utilities for comparing genomic features. Bioinformatics 26, 841-842 (2010).

106. Jensen, J. D., Foll, M. \& Bernatchez, L. The past, present and future of genomic scans for selection. Mol. Ecol. 25, 1-4 (2016).

107. Comstock, K. E. et al. Patterns of molecular genetic variation among African elephant populations. Mol. Ecol. 11, 2489-2498 (2002).

108. Fleischer, R. C., Perry, E. A., Muralidharan, K., Stevens, E. E. \& Wemmer, C. M. Phylogeography of the asian elephant (Elephas maximus) based on mitochondrial DNA. Evolution 55, 1882-1892 (2001).

109. Vidya, T. N. C., Sukumar, R. \& Melnick, D. J. Range-wide mtDNA phylogeography yields insights into the origins of Asian elephants. Proc. R. Soc. B Biol. Sci. 276, 893-902 (2009).

110. Gene Ontology Consortium. Gene Ontology Consortium: going forward. Nucleic Acids Res. 43, D1049-D1056 (2015).

111. Supek, F., Bošnjak, M., Škunca, N. \& Šmuc, T. REVIGO summarizes and visualizes long lists of gene ontology terms. PloS One 6, e21800 (2011).

112. Huang, D. W., Sherman, B. T. \& RA Lempicki. Systematic and integrative analysis of large gene lists using DAVID bioinformatics resources. Nat. Protoc. 4, 44-57 (2009).

113. Szklarczyk, D. et al. The STRING database in 2017: quality-controlled protein-protein association networks, made broadly accessible. Nucleic Acids Res. 45, D362-D368 (2017).

114. Weng, M.-P. \& Liao, B.-Y. modPhEA: model organism Phenotype Enrichment Analysis of eukaryotic gene sets. Bioinformatics 33, 3505-3507 (2017). 